\title{
Comparative Study of the Silver Nano-/Microstructures Deposited from Aerosol and Fog
}

\author{
A. V. Zheltova1, E. V. Maslyonkova², Yu. G. Biryukov³, P. R. Kasanskii ${ }^{4}$, \\ S. M. Ryndya ${ }^{5}$, A. S. Smolyanskii ${ }^{3,6}$
}

${ }^{1}$ Faculty of Bioengineering and Bioinformatics, Lomonosov Moscow State University, Moscow, Russia

${ }^{2}$ Pirogov Russian National Research Medical University, Moscow, Russia

${ }^{3}$ Branch of JSC "Karpov Institute of Physical Chemistry", Moscow, Russia

${ }^{4}$ JSC "Systems of Microscopy and Analysis", Moscow, Russia

${ }^{5}$ National Research Nuclear University "MePhi”, Moscow, Russia

${ }^{6}$ Mendeleev University of Chemical Technology, Moscow, Russia

Email: annettezv@gmail.com

How to cite this paper: Zheltova, A.V., Maslyonkova, E.V., Biryukov, Yu.G., Kasanskii, P.R., Ryndya, S.M. and Smolyanskii, A.S. (2017) Comparative Study of the Silver Nano-/Microstructures Deposited from Aerosol and Fog. Advances in Nanoparticles, 6, 128-139.

https://doi.org/10.4236/anp.2017.63011

Received: May 10, 2017

Accepted: August 7, 2017

Published: August 10, 2017

Copyright ( 2017 by authors and Scientific Research Publishing Inc. This work is licensed under the Creative Commons Attribution International License (CC BY 4.0).

http://creativecommons.org/licenses/by/4.0/

\begin{abstract}
A comparative study of the structure and fractal properties of arrays of the silver nano-/micro-particles deposited on the silicon substrate both from the aerosol and fog showed that the form of the silver individual particles and nano-/microstructures greatly depends on the deposition conditions. By passing an aerosol through isopropyl alcohol, the formation of fractal aggregates of the silver nano-/micro-particles both in the air and in alcohol was observed. Deposition of the silver nano-/micro-particles in the atmosphere of the saturated isopropyl alcohol vapours led to formation of fog. Microdroplets of the silver colloidal solution were deposited on the substrate. The further evaporation of alcohol created the silver nano/microstructures in the form of annular layers. It was found that the concerned annular layers contained silver particles of the same shape in the form of a Crescent (or Janus-nano-/microparticles). The nature of discovered effects is discussed.
\end{abstract}

\section{Keywords}

Aerosol, Fog, Silver, Deposition, Janus-Nanoparticle, Fractal Aggregate

\section{Introduction}

It is known [1], that anomalous optical properties of the metal nano-/microparticle (NMP) arrays can be determined by the interaction of light with the surface plasmon polaritons and localized plasmons. The structure and form of the me- 
tallic NMP are the important factors determining the efficiency of the concerned "plasmon-photon" interactions. Theoretical analysis [2] [3] [4] showed that the sickle shaped metallic NMP can enhance the interaction of localized plasmon with light photons, compared to the NMP having the spherical shape. However, the proposed method of the sickle metallic NMP synthesis by means of the colloidal microlithography method [5] is multi-stage, andrequires complicated hardware design. So the studies aim to simplify the method of the sickle metallic NMP synthesis.

The disadvantage of the newly proposed method of the metallic nano-/microstructures (NMS) layers synthesis by the "dry" aerosol deposition [6] consists in the random ballistic mechanism of coatings formation on the substrate surface. These coatings contain both the NMP of different sizes and the NMS of chain and dendritic shapes that can lead to significant scatter of the optical and other properties of the resulting porous metal films [7]. Therefore it is desirable to develop a method of the metallic NMS formation in the controlled conditions which could provide an opportunity for receiving the NMS of the same shape and, preferably, containing the arrays of the metallic NMP having the same geometric shape. Adherence to conditions allowed achieving the stability and reproducibility of the optical properties of the metallic NMS.

A promising approach to solve this problem may be thecreation of the metallic NMS precipitates formed during the evaporation of the micro-droplets from the metallic NMP colloidal solutions in various solvents [8] [9].

In the given case, depending on the conditions of the evaporation process [10], the metallic NMP deposits can exist either in the form of solid spots (this situation is considered in the "coffee stains" model [8]), or form of ring structure.

According to the research [8] [9] [10], the concerned method of the metallic NMS preparation can have many practical applications such as production of plasmon resonance sensors, catalytic surfaces, etc. The different forms of the received metallic NMS are due to the occurrence of the sedimentation processes in the highly non-equilibrium conditions which lead to the development of the self-organization. In that case the shape of the NMS deposits is determined by the deviation degree of the evaporation conditions from the equilibrium [10]. Usually the colloidal NMP solutions can be considered as the micro-emulsions which can be formed and stabilized by the surfactants. Then it is necessary to wash off the NMP sludge from the surfactant and emulsifier admixtures that can result in the deformation and the NMP damage. This problem can be solved if the deposition of the aerosol metallic particles on the silicone surface has been carried out in the atmosphere of the solute saturated vapors. Metallic NMP is the center where the condensation of the solute molecules and micro-droplet formation is taking place. Finally, the initial aerosol of the metallic particles transforms into fog [11]. After that, the evaporation of micro-droplets deposited on the silicone surface forms the metallic NMS, which do not contain the surfactant and emulsifier admixtures. 
The aim of present study consisted in:

i) the experimental confirmation of the possibility to form the ring metallic NMS synthesised by the evaporation of micro-droplets deposited on the substrate surface from the fog formed as a result of the interaction between the aerosol NMP and the solvent molecules;

ii) the study of the structure and shape of metal NMP contained in the NMS, which formed either after the micro-droplet deposition and evaporation, or the passing of aerosol NMP through the solvent media;

iii) conducting the fractal analysis of images that show the metal NMP shape and the structure of the metal NMS synthesized in both modes, obtained by transmission and scanning electron microscopy (TEM and SEM, respectively), and atomic force microscopy (AFM).

\section{Experimental}

1) To obtain the colloidal solutions of silver NMP in isopropyl alcohol (IPA) without the admixture of the emulsifier and surfactant molecules the self-made experimental facility [6] has been used (Figure 1(a)). The scheme of the concerned facilityincludes an aerosol spark generator, a diffusion aerosol spectrometer (DAS), the ultrasonic generator of the liquid and the aerosol flow stabilizer. After mixing the silver aerosol and the IPA saturated vapour the condensation processes were developed in the volume of the aerosol flow stabilizer and the micro-droplets of the colloidal solution appeared. Then these micro-droplets were deposited on the surfaces of three silicon wafers located at the output of the aerosol flow stabilizer. IPA vapours were obtained using the ultrasonic generator. For that purpose an IPA media with the chemical grade "pure for analysis" (State Standard 9805-84) was placed in ultrasonic bath before switching on the ultrasonic generator. Experiments were carried out at room temperature and at the atmospheric pressure. The IPA saturated vapour pressure was $\sim 25.3 \mathrm{~mm} \mathrm{Hg}$ [12], however not measured or fixed specifically. The silicon plates with dimensions $10 \times 10 \mathrm{~mm}$ and the thickness $0.5 \mathrm{~mm}$ (State Standard 8.592-2009) were used in research. The duration of the separateexperiment did not exceeded one hour.

The silver NMP aerosol was created with the help of the spark discharge between two silver electrodes and an air stream passed at the speed of $1 \mathrm{~cm} / \mathrm{s}$ through the working chamber of the aerosol spark generator [6]. The frequency of the spark breakdown was $50 \mathrm{~min}^{-1}$. Then the silver aerosol wastransported to the ultrasonic generator and to the DAS measuring system, which determined the size distribution and concentration of the silver NMP in the aerosol (Figure $1(b))$.

During the experiment the composition of the silver aerosol particles was controlledevery minute. It has been established that the largest part of the aerosol composition was about $45-50 \mathrm{~nm}$ (Figure 1(b)-1). The total number of the silver aerosol particles received in the volume of the working chamber during the experiment was about $\sim 5.2 \times 10^{6}$. As it is shown in Figure 1(b)-2, the particle 


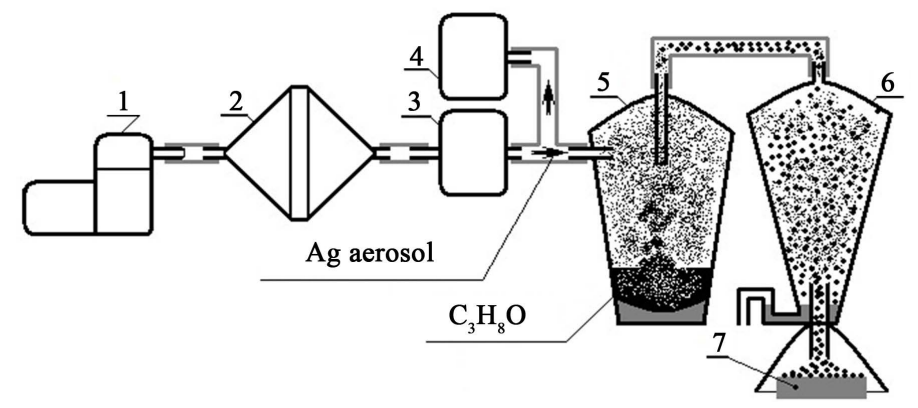

(a)

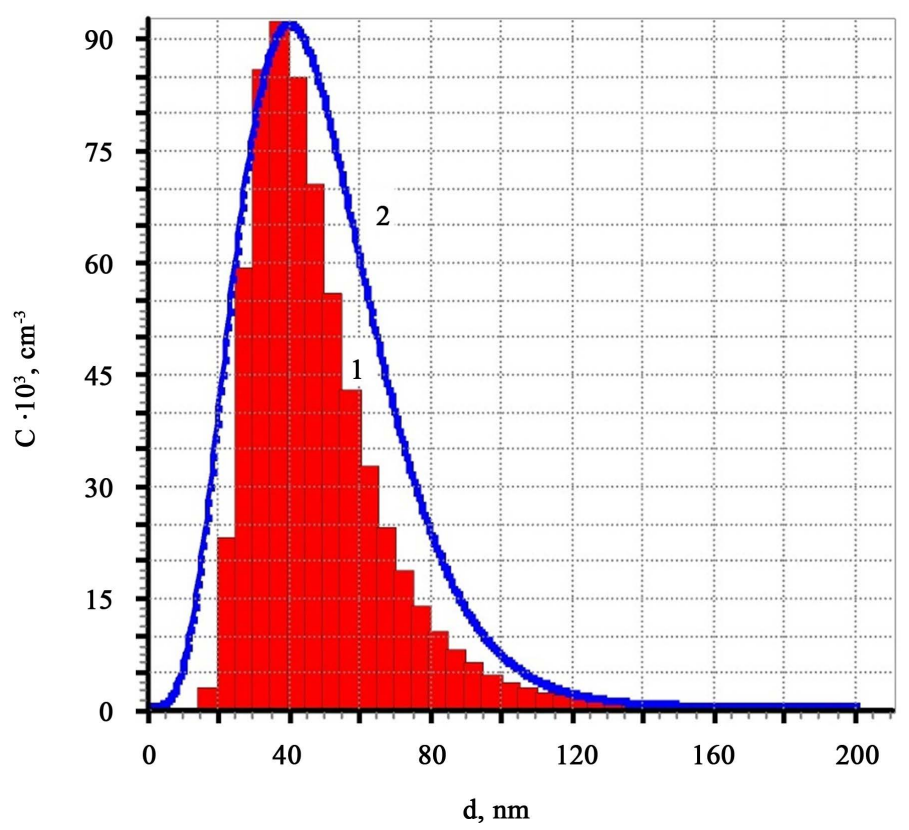

(b)

Figure 1. (a) Technological scheme of the silver NMP micro-droplets deposition: 1-micro-pump; 2-high efficiency particulate air filter; 3-aerosol spark generator; 4-diffusion aerosol spectrometer; 5-ultrasonic generator; 6-aerosol flow stabilizer; 7-silicon substrate. Black dots represent aerosol particles of silver; (b) Histogram (I) and the aerosol silver particle size distribution function (II) obtained by the approximation of experimental data by thelognormal distribution.

size distribution can be satisfactorily approximated by lognormal function [11] [13].

The colloidal solutions of silver NMP were also prepared by passing the aerosol through the IPA medium.

2) Before carrying out the TEM and SEM measurements the colloidal solutions of silver NMP in IPA were treated in an ultrasonic bath for 10 minutes. Then the sample of solution (about $\sim 0.1 \mathrm{ml}$ ) was deposited on the copper mesh modified by carbon.

Investigation of sludge formed on the silicon surface and carbon substrate after evaporation of the colloidal solutionofmicro-drops was conducted using TEM and SEM electronic microscopes FEI Tecna G2 F20 S-TwinTMP and Quanta-3D FEG (Figure 2 and Figure 3 correspondingly). TE-microscope FEI 

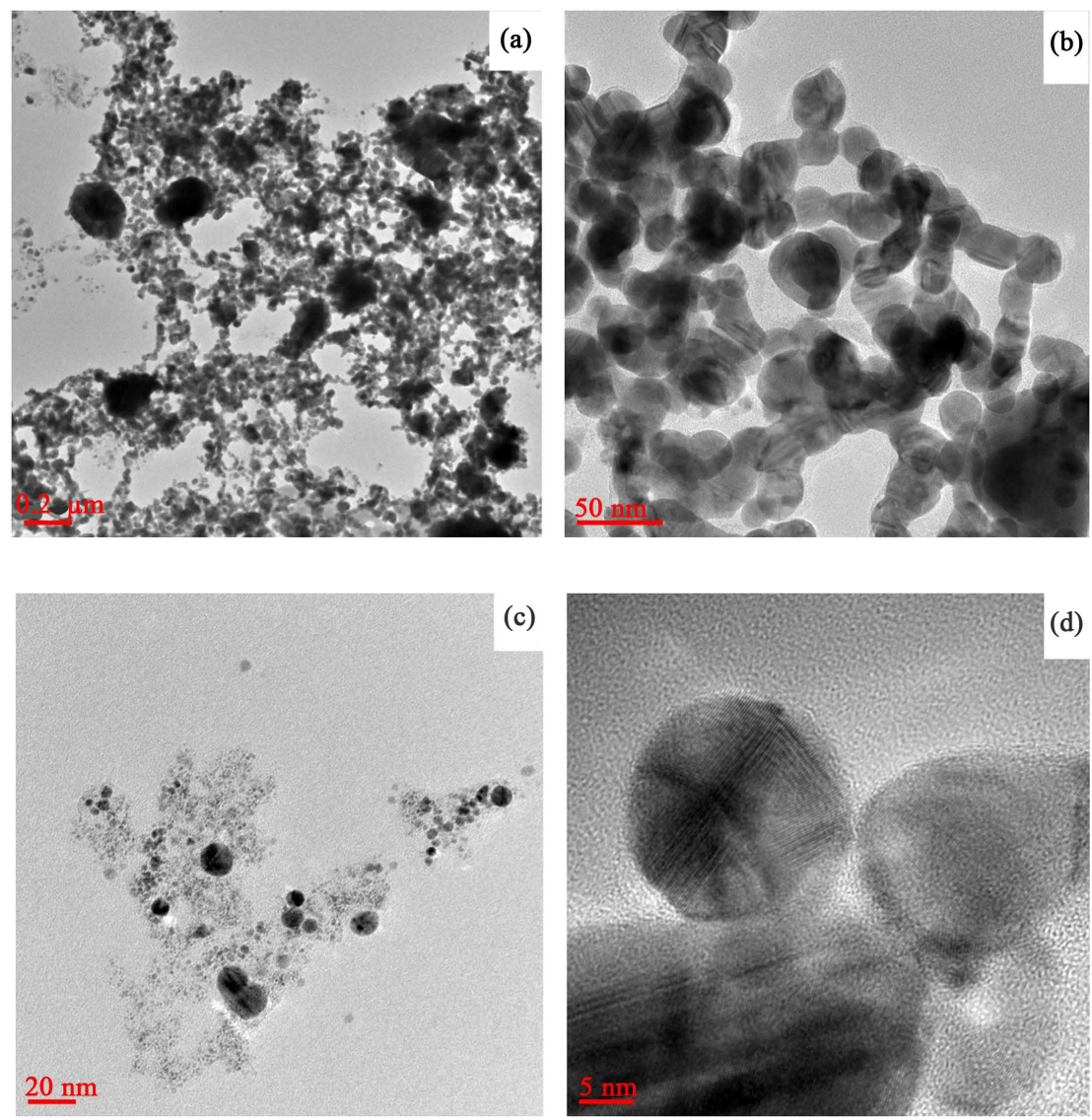

Figure 2. Images of the obtained silver nano-/microstructures on the copper-carbon substrate at different magnifications: $9900 \times(a) ; 38,000 \times(b) ; 97,000 \times(c)$; and 450,000× (d).

Tecna G2 F20 S-Twin TMP worked at an accelerating voltage of $200 \mathrm{kV}$ in bright field. The device resolution was $0.14 \mathrm{~nm}$ (line resolution).

It should be noted that the study of sludge which formed after evaporation of the micro-drops received by the second method was carried out during half an hour after it had beenplaced on a copper-carbon substrate. When the microdrops were received using the first method, the TEM- and SEM-measurements were performed after thestorage of the precipitates in the air and at the room temperaturewithin two weeks.

3) AFM investigation of the silicon surface roughness after deposition of the silver NMP arrays was carried out by Multimode 8 scanning probe microscope, manufactured by Bruker (USA).The concerned array of AFM data was further processed using Image J 1.49 [14] software with the help of the Fraclac plug-in [15].

\section{Results and Discussion}

\subsection{Silver Nano-/Microstructures Deposited from Aerosol}

As follows from the TEM images obtained in the first case the silver aerosol passage through IPA media resulted in intense NMP coagulation and formation of the different NMS types (Figure 2). In that case theformation of the numerous 

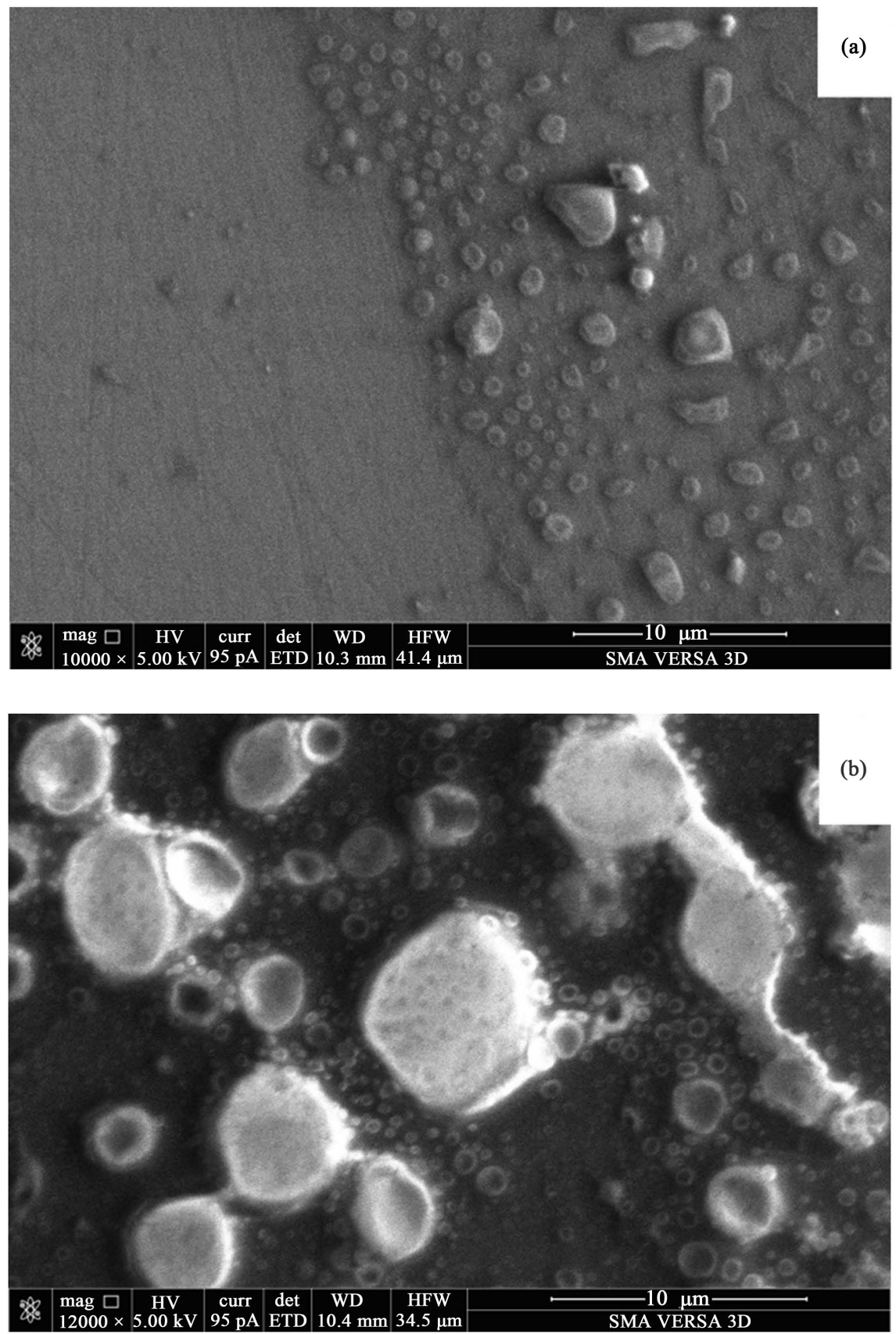

Figure 3. SEM-images of the silver nano-/microstructures on the silicon surface formed by evaporation of the micro-drops of colloidal solution. Measurement conditions are listed on the insert.

silver NMS resulted in th appearance of the porous silver film [6], that is an array of the randomly linked chains consisting of several (at least 10) silver NMP with sizes in the range of $10-20$ to $200 \mathrm{~nm}$ (Figures 2(a)-(c)). The facets of silver nano/microcrystals can be observed on the NMP surface (Figure 2(d)).

It is known [16], that the chain structure of NMS occurs as a result of the silver NMP coagulation in the air. Possibly, coagulation can take place in the IPA medium. The secondary structure observed in Figure 2(a) and Figure 2(b), consists of chaotically intertwined chains containing the numerous silver NMP's. 
Such structure occurs both at the stage of the storage in alcohol, and the evaporation of nano-/micro-drops. The emergence and growth of the secondary structures (or fractal aggregates [17]) isa result of the aggregationof the numerous silver particles in chains, and it can be described in terms of different models of aggregation [18]. The estimation of the fractal dimension for layers of the silver NMS located in the regions with high and low NMP concentrations (Figure 2(a) and Figure 2(b)) gives the values of the surface fractal dimension $\mathrm{D}=1.85$ and 1.99 which correspond to the case of Brownian motion and/or the motion of NMS's on a linear trajectory in the model of the three-dimensional "clustercluster association" [18]. Therefore, at the formation of the silver NMS layers which is described within of the "cluster-cluster association" model, the different mechanisms of silver particles fusion and structures can be implemented.

\subsection{Silver Nano-/Microstructures Deposited from Fog}

The observed ring shape of the silver NMS, synthesized by deposition from the fog, repeats the profile of evaporated micro-droplet (Figure 3). At the same timeit was found that the silver NMS's with sizes from 0.5 to $20 \mathrm{~nm}$ are uniformly distributed on the substrate surface that is consistent with the ideas of the "coffee stains" model [8] [9] [10].

Considering the arrays of the silver NMS, which are formed by the micro-droplets of colloidal silver solution in isopropyl alcohol on the silicon plate surfaces after deposition from fog (Figure 3(a) and Figure 3(b)). Despite the significant time interval between the deposition of the micro-droplets on the substrate and SEM-measurements, the border of drops can be clearly observed on the silicon surface. This may be due tothe sorption of the alcohol molecules by the silicon substrate. The numerous silver NMSs with the size from $<0.1$ to 3 $\mu \mathrm{m}$ can be observed on the SEM images also. It should be noted that a pronounced ring structure is characteristic for silver NMSs with the size from $<0.1$ to $1 \mu \mathrm{m}$.

According to [9] [10] [19], capillarity leads to one micron particle dynamics in evaporated drop. In this case, mass transfer processes can move the silver NMP either to the drop surface, or in the opposite direction depending on the ratio of the speed of the drop shape relaxation and the evaporation rate. The formation of the ring structure (or self-organization) may be considered a result of the mass transfer processes.

Unfortunately, due to the high level of the electron field emissionthe origin of which is associated with the adsorbed solvent molecules, it was impossible to carry out the SEM-studies of the ring silver NMS at the higher magnifications. Further investigation of the structure and distribution of silver NMPs in the ring boundary layer was carried out by AFM (Figure 4). As follows from the AFM-images (Figure 4(a) and Figure 4(b)), the size of the observed numerous silver NMP is about from 0.01 to $0.5 \mu \mathrm{m}$. All the silver particles considered have the same Crescent shape as well as the same orientation. All the observed silver NMPs are oriented by thickened side towards the outer border of the ring, while the Crescent is oriented towards the centre of the vaporized micro-droplet. 


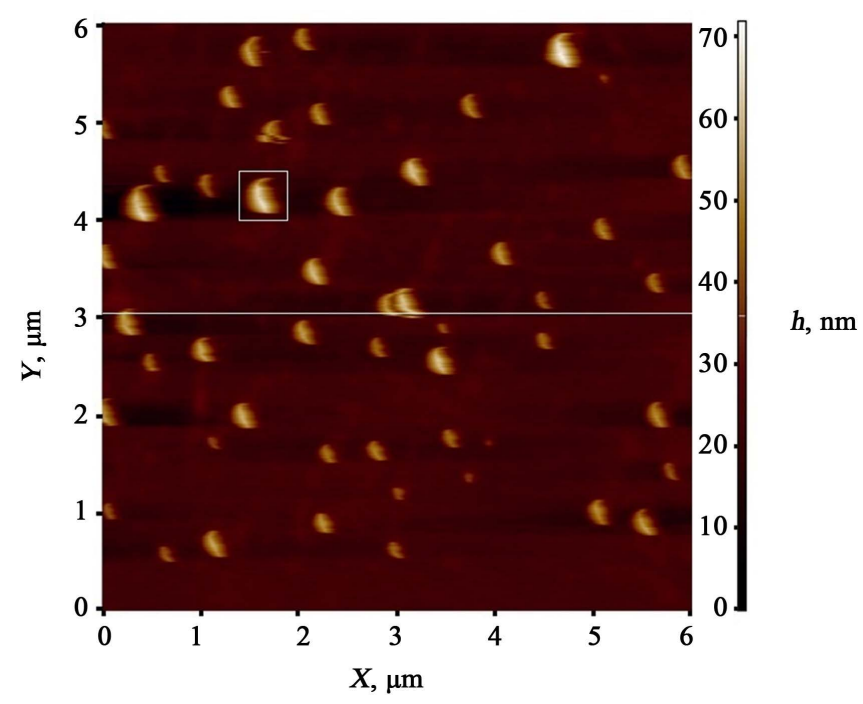

(a)

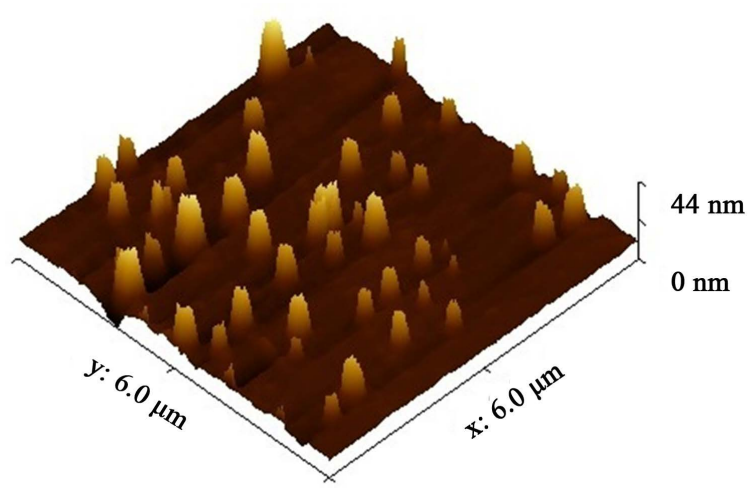

(b)

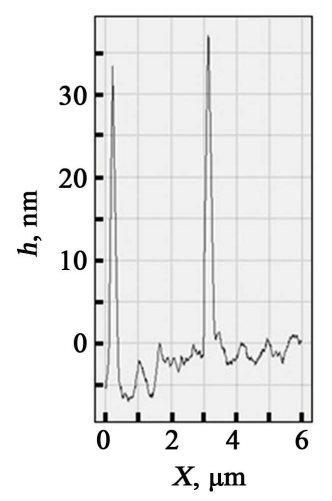

(c)

Figure 4. 2D- (a) and 3D-images (b) of the silver NMP arrays on the surface of the silicon support recorded by AFM-method in the field of the ring structure appeared in the place of the vaporized micro-droplets; (c) the AFM-profile of the silicon surface containing the silver nano-and micro-particles (shown by the white line on the image (a)). An enlarged image of the silver particle, selected by white rectangular frame, is shown in the Figure 5(a).

It is possible to result the following arguments in favour of the fact that with the help of AFM methodexactlythe silver particles have been registered. First, in the AFM profiles the silver NMP revealed itself as the fluctuations of the spatial distribution of the surface height with the magnitude from 30 to $35 \mathrm{~nm}$ (Figure 4(a) and Figure 4(c)), that is much higher than the surface roughness of the silicon substrate $(2.5-5.0 \mathrm{~nm})$. Second, the maximum height of the discovered sickle silver NMP is $44 \mathrm{~nm}$ that coincides with the maximum of the size distribution $(40-45 \mathrm{~nm})$ in the aerosol containing the silver NMP which has been determined previously (Figure 1(b)).

The most part of the sickle silver NMP's, shown in the Figure 4, has a two-or three-layer structure (Figure 5(a)). At least two aerosol particles participated in the formation of the considered silver NMP, which originally had a spherical shape (Figure 2(d)). Possibly, the deformation of the considered two-or three- 

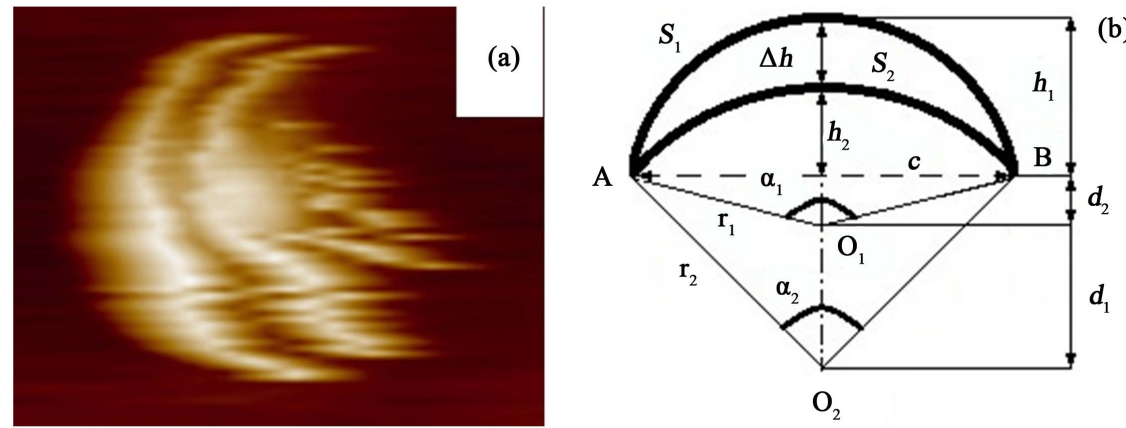

(b)

Figure 5. The image of the Crescent-shaped silver nano/microparticles (marked rectangle in Figure 4) obtained by atomic force microscopy (a) and the scheme of calculation the geometric parameters of the Crescent-shaped particles

particle clusters tends towards its boundaries away from the centre. Probably the mass-transfer processes have been moving in that direction during the evaporation of the IPA molecules.

An assessment of geometric characteristics of the Crescent-shaped silver NMP has been done. For this purpose, the shape of the particles has been represented in the form of a Crescent (Figure 5(b)) and the geometric parameters of the silver NMP have been calculated using the following formulas [20] [21]:

$$
\left\{\begin{array}{l}
h_{1}=h_{2}+\Delta h ; s_{i}=\alpha_{i} r_{i} ; r_{i}=h_{i}+d_{i}=\frac{h_{i}}{2}+\frac{c^{2}}{8 h_{i}} \\
c=\frac{2 r_{i} \sin \alpha_{i}}{2}=2 \sqrt{h_{i}\left(2 r_{i}-h_{i}\right)} ; \alpha_{i}=\frac{2 \arccos d_{i}}{r_{i}} \\
A_{i}=\frac{1}{2} r_{i}^{2}\left(\alpha_{i}-\sin \alpha_{i}\right)
\end{array}\right.
$$

In the system of the Equation (1) $i=1,2 ; h_{1}=h_{1}, h_{2}$ is the height of the circle segments, the arc lengths $s_{1}, s_{2}$ are part of these segments, correspondingly, limiting the considered particle with external and internal sides; $\Delta h$ is the thickness of the Crescent; $d_{1}, d_{2}$ are the distances from the centres of circles $\mathrm{O}_{1}$ and $\mathrm{O}_{2}$ to the middle of the chord $\mathrm{AB}$, which have the lengths $c, r_{1}, r_{2}$ are the radii of the circles with centres $\mathrm{O}_{1}$ and $\mathrm{O}_{2} ; \alpha_{1}, \alpha_{2}$ arethe angles $\mathrm{AO}_{1} \mathrm{~B}$ and $\mathrm{AO}_{2} \mathrm{~B} ; \mathrm{A}_{i}$ is the area of segments. The area of the Crescent is determined as the difference of the squares of segments.

According to the calculations, the radii of the circles $\mathrm{O}_{1}$ and $\mathrm{O}_{2}$ are practically identical (Table 1). Consequently, the Crescent-shaped silver particle is formed by two deformed aerosol NMP (Figure 5(a)). On the other hand, the estimated values of the circle radiiO $\mathrm{O}_{1}$ and $\mathrm{O}_{2}$ are 4 - 5 times higher than the most probable silver NMP size in the initial aerosol (Figure 1(b)). Probably, theobtained difference is due to the presence of a layer of the adsorbed isopropyl alcohol molecules, i.e. the cluster "silver NMP-isopropyl alcohol molecules" can be seen as a direct micelle. Possibly, the evaporation of microdroplets is accompanied by the coagulation of silver particles existing in the solution in the form of direct micelles.Further,clusters containing two or three NMP's, which surrounded by a layer of the IPA molecules have been created. 
Table 1. The geometrical characteristics of the Crescent modelling the silver sickle nano-/ micro-particles.

\begin{tabular}{cccc}
\hline \multirow{2}{*}{$№$} & Name, designation & \multicolumn{2}{c}{ Value } \\
\cline { 3 - 4 } & Arc 1 & Arc 2 \\
\hline $2^{*}$ & The thickness of the Crescent in the centre of the figure $\Delta h, \mu \mathrm{m}$ & 0.18 \\
$3^{*}$ & The length of the chord $c, \mu \mathrm{m}$ & 0.36 \\
4 & The height of the segment in the centre $h, \mu \mathrm{m}$ & 0.29 & 0.11 \\
5 & The radius of the circle $r, \mu \mathrm{m}$ & 0.201 & 0.205 \\
6 & The length of the arcs, $\mu \mathrm{m}$ & 0.82 & 0.44 \\
7 & Angle $\alpha$, degree & 232.69 & 125.72 \\
8 & The area of the segment $\mathrm{A}_{i}, \mu \mathrm{m}^{2}$ & 0.10 & 0.03 \\
\hline
\end{tabular}

Note: $\left({ }^{*}\right)$ Thevalues are determined by measuring the Figure $5(\mathrm{a})$.

The processes of spinodal decomposition [22] of the considered clusters and the formation in the cluster volume of the two-phase system containing the layer of the deformed Crescent shape silver NMPs have been developed during further drying and storage of the sample. Subsequent evaporation of the IPA molecules leads to the formation of the observed silver Crescent-shaped NMP. It is known [23], that this phenomenon is often observed in the study of the metastable heterogeneous colloidal systems as a result of the complex thermo- and masstranfer processes [24] and it is called "the syneresis".

According to [25] [26] [27] the driving force the effect of syneresis is concentration gradient of the alcohol molecules in the cluster and on the substrate surface during the evaporation process. The value of the occurring stress concentration is sufficient for cluster splitting, and deformation of the original spherical silver NMP's.

The value of the fractal dimension for silver Crescent-shaped NMP (Figure 5 (a)) is about of $\mathrm{D} \sim 1.69$, that is significantly lower than the established similar values for the silver NMP fractal dimension obtained by passing the silver aerosol though the IPA medium [18]. This value of the fractal dimension D corresponds to the model of the "cluster-cluster association in the regime of Brownian motion model" [18].

\section{Conclusions}

Thus, even a slight change in the conditions of synthesis NMP arrays can radically affect the shape of the precipitate and the structure of the silver particles. Probably, the formation of the precipitate and the shape of silver NMP can be affected by other factors, namely: the surface roughness of the substrate, the temperature and the pressure of alcohol vapour, humidity, and so on. Establishing the role of the considered factors, as well as the creation of the micro-droplet evaporation model, which can explain the nature of the discovered effects, are subject for further researches. 
Thus, experimentally synthesized silver NMS with sickle NMP's was obtained by means of controlled evaporation of the colloidal solutions droplets deposited from the fog on the surface of the silicone support. It is possible that the arrays of the silver Crescent-shaped NMP's may be associated with the processes of silver nanoparticles self-organization, which develop during the micro-drops evaporation. In conclusion, it should be noted that the detected silver Crescentshaped NMP belongs to the recently discovered kind of specific asymmetric nanoparticles-the so called Janus silver nanoparticles [25] [26] [27].

\section{Funding}

The present study was performed with the financial support of the ministry of education and science of the Russian federation (Subsidy No. 14.576.21.0053 to conduct applied research from October 20, 2014, unique identifier RFMEFI57614X0053) as well as Russian fund of basic research (project No. 17-07-00524).

\section{References}

[1] Klimov, V.V. (2009) Nanoplasmonics. Fizmatlit, Moscow, 480 p. (in Russian)

[2] Aubry, A., Dang, Y.L., Maier, S.A. and Pendry, J.B. (2010) A Broadband Plasmonic Device Concentrating the Energy at the Nanoscale: The Crescent-Shaped Cylinder. Physical Review, 82B, 125430.

[3] Bocchio, N., Alvarez, M., Gaul, F., Rochholz, H., Koynov, K. and Kreiter, M. (2007) Crescent-Shaped Nanoparticles: Optical Properties and Applications. Molecular Plasmonics. International Symposium, Abstracts. Jena, 10-12 May 2007.

[4] Krapek, V., Koh, A.L., Brinek, L., Hrton, M., Tomanec, O., Kalousek, R., Maier, S.A. and Sikola, T. (2015) Spatially Resolved Electron Energy Loss Spectroscopy of Crescent-Shaped Plasmonic Antennas. Optics Express, 23, 11855 https://doi.org/10.1364/OE.23.011855

[5] Rochholz, H., Bocchio, N. and Kreiter, M. (2007) Tuning Resonances on Crescent-Shaped Noble-Metal Nanoparticles. New Journal of Physics, 9, 53. https://doi.org/10.1088/1367-2630/9/3/053

[6] Smolyanskii, A.S., Zagaynov, V.A., Biryukov, Yu.G., Magomedbekov, E.P., Stepanova, O.G. and Trakhtenberg, L.I. (2014) The Structural Properties of the Layers of Silver Nano-/Microparticles Synthesized on the Surface of Nuclear Filters by the Method of Aerosol Spraying. Composites and Nanostructures, 6, 137-147. (in Russian)

[7] Gaponenko, S.V., Rosanov, N.N., Ivchenko, E.L., Fedorov, A.V., Baranov, A.V., Bonch-Bruevich, A.M., Vartanyan, T.A. and Przybelski, S.G. (2005) In: Fedorov, A.V., Ed., Optics of Nanostructures, Nedra, St. Petersburg, 326 p. (in Russian)

[8] Layani, M., Gruchko, M., Milo, O., Balberg, I., Azulay, D. and Magdassi, S. (2009) Transparent Conductive Coatings by Printing Coffee Ring Arrays Obtained at Room Temperature. ACS Nano, 3, 3537-3542. https://doi.org/10.1021/nn901239z

[9] Vysotskaya, V.V., Uryupin, O.J., Senchikhin, I.N. and Roldugin, V.I. (2013) Formation of a Ring of Precipitation When Evaporation of Drops of the Dispersions of Silver Nanoparticles and Studies of the Fine Structure. Colloidal Journal, 75, 161-169. (in Russian)

[10] Vodolazsky, I.V., Tarasevich, Yu.Yu. and Isakova, O.P. (2010) Simulation of the 
Evolution of the Phase Front on a Horizontal Substrate Drying a Drop of Colloidal Solution. Nonlinear World, 8, 142-150. (in Russian)

[11] Reist, P. (1997) Aerosols. Mir, Moscow.

[12] Kutateladze, S.S. and Borishanskii, V.M. (2012) Handbook of Heat Transfer. Book on Demand, Moscow.

[13] Lee, K.W. and Chen, H. (1984) Coagulation Rate of Polydisperse Particles. Aerosol Science and Technology, 3, 327-334. https://doi.org/10.1080/02786828408959020

[14] Ferreira, T. and Rasband, W. (2012) ImageJ User Guide-IJ 1.46r. http://imagej.nih.gov/ij/docs/guide

[15] Karperien, A.L. (2013) Fraclac for ImageJ. Charles Sturt University, Albury-Wodonga. 1-36.

[16] Aloyan, A.E., Arutyunyan, V.O., Lushnikov, A.A. and Zagaynov, V.A. (1997) Transport of Coagulating Aerosol in the Atmosphere. Journal of Aerosol Science, 28, 67-85. https://doi.org/10.1016/S0021-8502(96)00043-2

[17] Kolesnichenko, A.V. and Marov, M.Y. (2014) Modeling of Processes of Formation of Dust Fractal Clusters as the Basis of Loose Preplanetesimals in the Solar Deplanatum the Cloud. Preprint IPM, Moscow. http://library.keldysh.ru/preprint.asp?id=2014-75

[18] Smirnov, B.M. (1986) Fractal Clusters. Uspekhi Fizicheskih Nauk, 149, 177-219. https://doi.org/10.3367/UFNr.0149.198606a.0177

[19] Suzdalev, I.P. (2006) Nanotechnology: Physico-Chemistry of Nanoclusters, Nanostructures and Nanomaterials. Komkniga, Moscow.

[20] Korn, G. and Korn, T. (1977) Mathematics Handbook (for Science Officers and Engineers). Nauka, Moscow.

[21] Online Calculator. http://planetcalc.ru

[22] Rempel, A.A. and Valeeva, A.A. (2015) Materials and Methods of Nanotechnology. Publishing House Ural. University Press, Ekaterinburg.

[23] Fridrikhsberg, D.A. (1995) Course of Colloid Chemistry. Khimiya, St. Petersburg.

[24] Geguzin, J.E. (1984) Physics of Sintering. Nauka, Moscow.

[25] Olenin, A.Y., Nizamov, T.R. and Lisichkin, G.V. (2014) Chemical Modification of the Surface of Silver Nanoparticles. Obtaining Janus-Particles. Russian Nanotechnology, 9, 19-24.

[26] Song, Y. and Chen, S.W. (2014) Janus Nanoparticles: Preparation, Characterization, and Applications. Chemistry-An Asian Journal, 9, 418-430. https://doi.org/10.1002/asia.201301398

[27] Lattuadaa, M. and Hattonb, T.A. (2011) Synthesis, Properties and Applications of Janus Nanoparticles. Nano Today, 6, 86-308. https://doi.org/10.1016/j.nantod.2011.04.008 
Submit or recommend next manuscript to SCIRP and we will provide best service for you:

Accepting pre-submission inquiries through Email, Facebook, LinkedIn, Twitter, etc. A wide selection of journals (inclusive of 9 subjects, more than 200 journals)

Providing 24-hour high-quality service

User-friendly online submission system

Fair and swift peer-review system

Efficient typesetting and proofreading procedure

Display of the result of downloads and visits, as well as the number of cited articles Maximum dissemination of your research work

Submit your manuscript at: http://papersubmission.scirp.org/

Or contact anp@scirp.org 